This can be achieved by the evaporation of water into warm air using high pressure fogging or evaporative pad systems. When selecting a heat abatement system, one must consider production goals, breeding facilities (closed or semi-open buildings, water supply) and climatic environment (temperature and relative humidity). While differences in thermal-tolerance exist between livestock species (ruminants $>$ monogastrics), there are also large differences between breeds of a species and within each breed. Consequently, the opportunity may exist to improve thermal tolerance of the animals using genetic tools. Generally, most probably genes that are involved in acclimation to heat stress are also involved in development of thermal tolerance. The adaptive capacities of tropical breeds are generally related to an improved ability to lose heat (hair coat characteristics, special appendices...) and/or to a reduced metabolic HP due to their lower production level or small size. These characteristics could be used to improve thermal tolerance in commercial breeds using crossbreeding strategies or gene introgression. Selection based on rectal temperature measurements and inclusion of a THI in the genetic evaluation models are promising in pigs and dairy cattle. However, further research is needed to quantify the genetic antagonism between adaptation and production traits to evaluate the potential selection response. With the development of molecular biotechnologies, new opportunities are available to characterize gene expression and identify key cellular responses to heat stress. These new tools will enable to improve the accuracy and the efficiency of selection for heat tolerance. Epigenetic regulation of gene expression and thermal imprinting of the genome could also be an efficient method to improve thermal tolerance. Such techniques (e.g. perinatal heat acclimation) are currently being studied in chicken.

In conclusion, a variety of technologies can be used to alleviate heat stress and improve production level under hot climate. The ability of the producers to access to these technologies will depend on availability and prices of water and energy and on the livestock production system (intensive vs. extensive).

\title{
Physiology of adaptation to nutritional stress in farm animals: from production characterization to proteome profiling
}

\author{
André Almeida ${ }^{\dagger}$ and Luís Alfaro Cardoso \\ Instituto de Investigação Científica Tropical \& Centro Interdisciplinar de Investigação em Sanidade Animal. CVZ-FMV, Av. Univ. Técnica, 1300-477 Lisboa, Portugal
}

\begin{abstract}
Introduction
Farm animals have adapted to the constraints of their production systems. Seasonal weight loss (SWL) is the major constraint to animal production in tropical regions (Almeida and Cardoso, 2008). Coupled to production studies, the study of the metabolic changes to food restriction, highlighting energy and protein metabolic saving mechanisms, can be a useful approach to identify the physiological pathways relevant in breed selection and to identify genetic biomarkers that could be used in animal selection. Our research has been focused on several aspects related to weight loss. Here we present major results and conclusions obtained.
\end{abstract}

\section{Weight Loss, serum amino acid and myofibrillar protein profiling in Boer goats}

(Almeida et al., 2004, 2006) The aim of the work was to determine serum free amino acid and 1D gel electrophoresis myofibrillar protein (skeletal muscle) profiles in Boer goat bucks following protein and energy undernutrition (20\% weight loss). Control animals showed higher concentrations ( $\mu \mathrm{mol} \mathrm{L}^{-1}$ ) of Ala, Tyr and Cit, whilst the restricted group showed higher concentration of Val, Ile, Leu, Thr, Met, Lys, Tau, Orn, Hyp and 3-metyl histidyne (Me3His), while Gly, Ser, Asp, Glu, Arg, His and Pro were similar in both groups. The restricted group showed myofibrillar protein degradation of protein $\mathrm{C}$ and $\alpha$-actinin. It can be concluded that the degradation of small carbon chain amino acid has a higher efficiency than degradation of long carbon chain amino acid and a disruption of muscle structure at the level of the $A$ band (protein $C$ ) and the matrix of the $Z$ line ( $\alpha$-actinin).

\section{Weight Loss and skeletal muscle proteomics profiling in the rabbit}

(Almeida et al., 2010) In this study, a 20\% weight reduction was induced in two rabbit breeds: NZW - New Zealand White, a meat producer, and wild rabbit, aiming to determine differential protein expression in the skeletal muscle within control (ad libitum) and restricted diet animals, using two-dimensional gel electrophoresis (2DE) and peptide mass fingerprinting. Results show that L-lactate dehydrogenase, adenylate kinase, enolase, aldolase and glyceraldehyde 3-phosphate dehydrogenase, which are enzymes involved in energy metabolism, are differentially expressed in the restricted diet experimental animal groups. Breed differences were also noticed since NZW rabbits show higher expression of structural proteins. These proteins are available to be further tested as relevant biomarkers of weight loss and objects of manipulation as a selection tool towards increasing tolerance to SWL.

\footnotetext{
${ }^{\dagger}$ E-mail: aalmeida@fmv.utl.pt
} 


\title{
Weight loss and liver proteomics profiling in three tropical sheep breeds
}

The objective was to determine differential protein expression in the liver of three different sheep breeds of sheep that show different levels of adaptation to SWL: Australian Merino (highly susceptible), Damara (tolerant) and Dorper (intermediate). Liver samples were obtained from both control and underfed, weight-losing animals of the three breeds using two-dimension electrophoresis and identification by mass spectrometry. Expression patterns of Gluthathione S-transferase (merino groups only), carbonic anhydrase 3 (2 fold increase of expression in control animals) and carbonyl reductase (present in all groups except Damara underfed and Dorper control) are of interest as a consequence of their role: detoxification of endogenous compounds and acid/base balance, respectively.

\section{Concluding Remarks}

Weight loss significantly affects production parameters and biochemical/physiological profiles in domestic animals. The study on how does SWL affect Nitrogen metabolism plays a key role in farm animal selection, particularly concerning adaptation to SWL. Our research has established several biomarkers of tolerance/adaptation to weight loss that in conjunction with genomics and transcriptomics will play an important role in selection.

\section{Acknowledgements}

Authors thank FCT for research funding. Contributions of JPC greyling, LM Schwalbach, HO de Waal (UFS, Bleomfontein, South Africa), the T Kilminster, T Scanlon, J Greef and C Oldham (DAFWA, Perth, Australia) and A Campos, R Francisco, AV Coelho (ITQB, Portugal) are acknowledged.

\author{
References \\ Almeida AM, Campos A, Francisco R, van Harten S, Cardoso LA and Coelho AV 2010. Animal Genetics 41, $260-272$. \\ Almeida AM and Cardoso LA 2008. Tropical Animal Health and Production 40, 529-536. \\ Almeida AM, Schwalbach LM, de Waal HO, Greyling JPC and Cardoso LA 2004. Small Ruminant Research 55, 141-147. \\ Almeida AM, Schwalbach LM, de Waal HO, Greyling JPC and Cardoso LA 2006. Tropical Animal Health and Production 38, $443-449$.
}

\section{Thermoregulatory response and relationships with performance of Large White growing pigs reared in tropical humid climate}

\author{
Jean-Luc Gourdine ${ }^{\dagger}$, Maryse Cyrille and David Renaudeau
}

INRA, UR143, Unité de Recherches Zootechniques, 97170 Petit Bourg, Guadeloupe, France

\begin{abstract}
Introduction
Ambient temperature $(T)$ is one of the factors affecting performance of pigs. When $T$ is elevated, growing pigs reduces metabolic heat production by decreasing their voluntary feed intake in order to maintain homoethermia (Renaudeau et al., 2004). Several studies show the consequences of heat stress in growing pigs (Collin et al., 2000; Quiniou et al., 2000). However, most of these studies were performed in controlled conditions with two or several levels of T. Studies performed in semi-open buildings subjected to the natural changes of tropical climate are rare. This study was conducted to evaluate the thermoregulatory responses and their relationships with performance and feeding behaviour in a total of 63 Large White growing pigs reared during the warm season in a tropical humid climate. The study was also carried out to evaluate to what extent the phenotyping of heat tolerance in pigs is routinely feasible in semi-open conditions.
\end{abstract}

\section{Materials and Methods}

The data used concerned a contemporary group of 63 Large White growing pigs (33 females and 30 barrows), raised at the INRA experimental herd of Duclos in Guadeloupe ( $16^{\circ} \mathrm{N}$ latitude, $61^{\circ} \mathrm{W}$ longitude). The data covered the BW range from $26.9 \pm 3.6$ to $84.2 \pm 9.1 \mathrm{~kg}$ (mean \pm SD) from March to May 2010. Thirty-two pigs housed in rooms with automatic feed dispensers (DAC) (4 rooms with 8 pigs each) allowing to feed pigs and to measure their feeding behavior automatically; 31 other pigs were fed together (3 rooms with 10-11 pigs). The rectal temperature (RT) and the cutaneous temperature (CT) were measured twice daily (between 07:00 and 09:30 and between 12:00 and 14:30), every 15 days until the end of growth. Body weight (BW) and lengths of the back were measured in the morning. Ambient

\footnotetext{
${ }^{\dagger}$ E-mail: Jean-Luc.Gourdine@antilles.inra.fr
} 